\title{
GLR TESTS FOR FAULT DETECTION OVER SLIDING DATA WINDOWS
}

\author{
David Törnqvist* Fredrik Gustafsson* Inger Klein *
}

\author{
* Department of Electrical Engineering \\ Linköpings universitet, SE-58183 Linköping, Sweden \\ E-mail: \{tornqvist, fredrik, inger\}@isy.liu.se
}

\begin{abstract}
The Generalized Likelihood Ratio (GLR) test for fault detection as derived by Willsky and Jones is a recursive method to detect additive changes in linear systems in a Kalman filter framework. Here, we evaluate the GLR test on a sliding window and compare it to stochastic parity space approaches. Robust fault detection defined as being insensitive to faults in the signal space is also studied in the GLR framework.
\end{abstract}

Keywords: fault detection, statistical signal processing, robust estimation, parity space

\section{INTRODUCTION}

The work concerns primarily linear Gaussian models, which over a sliding window can be represented by the following signal model

$$
Y=\mathcal{O} x+H_{u} U+H_{v} V+H_{f} F+E,
$$

where $Y$ is a vector of outputs during a sliding window time period, $x$ is the initial state of the system, $\mathcal{O}$ is the extended observability matrix, $H_{u}, H_{v}$ and $H_{f}$ are Toeplitz matrices describing how the inputs, $U$, process noise, $V$, and faults, $F$, enter the system and $E$ is additive measurement noise. The sum of two noise terms is a Gaussian noise with $\operatorname{Cov}\left(H_{v} V+\right.$ $E)=S$. The details are described in Section 2 and in (Gustafsson 2001). Fault detection is here seen as the hypothesis test

$$
\begin{array}{ll}
H_{0}: & F=0 \\
H_{1}: & F \neq 0 .
\end{array}
$$

Recently, (Desai and Mangoubi 2003) proposed robust fault detection as only testing for faults that do not lie in the signal space. In our notation, the signal space is spanned by the observability matrix, which means that we exclude faults $F$ such that $H_{f} F$ belongs to the column space of $\mathcal{O}$ and thus can be explained by a different state vector. Using the pseudo-inverse $H_{f}^{\dagger}$, this means that $F$ does not belong to the range of $H_{f}^{\dagger} \mathcal{O}$ and the hypothesis test becomes

$$
\begin{array}{ll}
H_{0}: & F \in \mathcal{R}\left(H_{f}^{\dagger} \mathcal{O}\right) \\
H_{1}: & F \notin \mathcal{R}\left(H_{f}^{\dagger} \mathcal{O}\right),
\end{array}
$$

where $\mathcal{R}(\cdot)$ denotes the range. These two tests will be treated in parallel.

The basic residual to use in fault detection is $r=Y-$ $H_{u} U$, which depends on the state $x$. The classic parity space approach (Basseville and Nikiforov 1993, Chow and Willsky 1984, Ding et al. 1999, Gertler 1997, Gertler 1998), computes a projection of this residual to the null space of the observability matrix, so $W_{1}^{T} r=$ $W_{1}^{T}\left(H_{f} F+H_{v} V+E\right)$ is independent of the state. The original parity space approach is a deterministic one, so this residual is non-zero for non-zero faults. The extension to process and measurement noise was done in (Gustafsson 2002), and analytical results on detectability of an all-one fault vector $F$ was derived.

The first extension here is to arbitrary fault vectors, including incipient faults (slowly increasing) and general time-varying fault profiles. The GLR approach (Willsky and Jones 1976) maximizes the likelihood ratio function over all faults, and the explicit GLR test statistic will be given. It turns out that this in both 
hypotheses tests (2) and (3) can be expressed as a certain projection of the residual.

A second approach is to estimate the state rather than projecting the residual to the parity space.

\section{NOTATION}

\subsection{State space model to signal model}

The linear system is defined as the state space model

$$
\begin{aligned}
x_{t+1} & =A_{t} x_{t}+B_{u, t} u_{t}+B_{f, t} f_{t}+B_{v, t} v_{t} \\
y_{t} & =C_{t} x_{t}+D_{u, t} u_{t}+D_{f, t} f_{t}+e_{t}
\end{aligned}
$$

We separate the following types of input:

- Deterministic known input $u_{t}$. This is common in control applications.

- Deterministic unknown fault input $f_{t}$, which is used in the fault detection literature. The known matrices $B_{f, t}$ and $D_{f, t}$ determines which part of the system will be affected by the different faults.

- Stochastic unknown disturbances $v_{t}$ and $e_{t}$, process noise and measurement noise, respectively, which are used in the Kalman filter setting. Both will here be assumed to be independent and Gaussian, with zero mean and covariance matrices $Q_{t}$ and $R_{t}$, respectively.

To establish the correspondence of models (4) and (1), stack $L$ signal values to define the signal vectors $Y_{t}=\left(y_{t-L+1}^{T}, \ldots, y_{t}^{T}\right)^{T}$, etc for all signals. We here use the time index $t$ to note that fault detection is a recursive task. Also define the Toeplitz matrices (time indices are omitted for simplicity)

$$
H_{s}=\left(\begin{array}{cccc}
D_{s} & 0 & \cdots & 0 \\
C B_{s} & D_{s} & \cdots & 0 \\
\vdots & & \ddots & \vdots \\
C A^{L-2} B_{s} & \cdots & C B_{s} & D_{s}
\end{array}\right)
$$

for all signals $s=u, f, v$ and the observability matrix

$$
\mathcal{O}=\left(\begin{array}{c}
C \\
C A \\
\vdots \\
C A^{L-1}
\end{array}\right)
$$

Equation (4) can then be written as

$$
\begin{aligned}
Y_{t}-H_{u} U_{t} & = \\
& \mathcal{O} x_{t-L+1}+H_{f} F_{t}+H_{v} V_{t}+E_{t} .
\end{aligned}
$$

which is (1). Note that we use the notation $x$ for $x_{t-L+1}$ to shorten the notation from here on. The definition of

$$
S=\operatorname{Cov}\left(H_{v} V_{t}+E_{t}\right)
$$

is straightforward.

\subsection{Basic definition and mathematical tools}

The basic tools in the derivation are the following:

- The pseudo-inverse operation used here is the Moore-Penrose inverse which can be found in, i.e., (Golub and van Loan 1996). In the case where $A$ has full row rank it can be computed as $A^{\dagger}=\left(A^{T} A\right)^{-1} A^{T}$.

- Projection operator. A projection on the range space, $\mathcal{R}(A)$, spanned by the columns in $A$ is given by $\mathcal{P}_{A}=A\left(A^{T} A\right)^{-1} A^{T}=A A^{\dagger}$, with the obvious property $\mathcal{P}_{A} A=A$. $\mathcal{R}_{A}$ denotes a basis for $\mathcal{R}(A)$.

- Projection on null space. To remove the state dependence in (1), the orthogonal projection $I-$ $\mathcal{P}_{\mathcal{O}}$ is used, with the obvious property ( $I-$ $\left.\mathcal{P}_{\mathcal{O}}\right) \mathcal{O}=0 . \mathcal{N}_{\mathcal{O}}$ is a basis for the null space of the columns in $\mathcal{O}$ denoted $\mathcal{N}(\mathcal{O})$.

- Whitening. Assume that $\operatorname{Cov}(r)=P$, then $\operatorname{Cov}\left(P^{-1 / 2} r\right)=I$, so pre-multiplying with $P^{-1 / 2}$ is a whitening operation. A normalized residual can then be defined as $\bar{r}=P^{-1 / 2} r$.

- Minimum variance (MV) estimation. For the equation system $A x=r$, the least squares (LS) solution $\hat{x}^{L S}=A^{\dagger} r$ is the minimum variance estimate if and only if $\operatorname{Cov}(r)=I$. That is, using pre-whitened residual, we have

$$
\begin{aligned}
\hat{x}^{M V}=\left(P^{-1 / 2} A\right)^{\dagger} P^{-1 / 2} r & \\
& =\left(A^{T} P^{-1} A\right)^{-1} A^{T} P^{-1} r .
\end{aligned}
$$

- GLR test. To test whether $F_{t}=0$ or not when $\bar{r} \sim \mathrm{N}\left(P^{-1 / 2} H_{f} F_{t}, I\right)$, the log likelihood ratio of the two hypotheses is first formed:

$$
\begin{gathered}
L\left(F_{t}\right)=2 \log \frac{e^{-\frac{1}{2}\left\|\bar{r}-P^{-1 / 2} H_{f} F_{t}\right\|_{2}^{2}}}{e^{-\frac{1}{2}\|\bar{r}\|_{2}^{2}}}= \\
=\|\bar{r}\|_{2}^{2}-\left\|\bar{r}-P^{-1 / 2} H_{f} F_{t}\right\|_{2}^{2} .
\end{gathered}
$$

The likelihood ratio is then maximized over the unknown parameter $F_{t}$ to get the GLR test statistic

$$
\begin{gathered}
L=\max _{F_{t}}\left(\|\bar{r}\|_{2}^{2}-\left\|\bar{r}-P^{-1 / 2} H_{f} F_{t}\right\|_{2}^{2}\right)= \\
=/ F_{t}=\left(P^{-1 / 2} H_{f}\right)^{\dagger} \bar{r} /= \\
=\left(\|\bar{r}\|_{2}^{2}-\left\|\bar{r}-P^{-1 / 2} H_{f}\left(P^{-1 / 2} H_{f}\right)^{\dagger} \bar{r}\right\|_{2}^{2}\right) \\
=\left(\bar{r}^{T} \bar{r}-\bar{r}^{T}\left(I-\mathcal{P}_{P^{-\frac{1}{2} H_{f}}}\right) \bar{r}\right)=\bar{r}^{T} \mathcal{P}_{P^{-\frac{1}{2} H_{f}}} \bar{r}
\end{gathered}
$$

The test statistic, $L$, is a sum of squared Gaussian variables with $\operatorname{rank}\left(\mathcal{P}_{P^{-1 / 2} H_{f}}\right)$ degrees of freedom. It is therefore $\chi^{2}\left(\operatorname{rank}\left(\mathcal{P}_{P^{-1 / 2} H_{f}}\right)\right)$ distributed. The assumption here is that $\bar{r}$ has uncorrelated elements and $\operatorname{dim}(\bar{r}) \geq \operatorname{rank}\left(\mathcal{P}_{P^{-1 / 2} H_{f}}\right)$. Based on knowledge about the distribution of the test statistic a suitable threshold for detection can be chosen.

- Fault profile model. To get a low order parameterization of the fault profile, and a nonambigous distinction of fault and process noise, assume that the fault profile is a smooth function 
(rather than noise). That is, Let $f_{t}=F m_{t}$, where $F$ defined a certain fault direction, and $m_{t}$ is the scalar time-varying magnitude. Choose basis functions $\varphi_{t}$ of smooth functions (for instance polynomials), so that we get a model $m_{t}=\varphi_{t}^{T} \theta$. For simplicity, assume an orthonormal basis (for instance Legendre polynomials), such that $\sum_{k=t-L+1}^{t} \varphi_{t} \varphi_{t}^{T}=I$. In that case, we preserve fault energy so $\left\|m_{t}\right\|^{2}=\sum_{k=t-L+1}^{t} m_{t}^{2}=$ $\|\theta\|^{2}$. Then, use $\bar{B}_{f, t}=B_{f, t} F \varphi_{t}^{T}$ and $\bar{F}=\theta$ in (4), which gives $F_{t}=\theta$ in (7).

- Residual with uncorrelated elements. Define a prediction error as

$$
\varepsilon=Y-H_{u} U-\mathcal{O} \hat{x} .
$$

The prediction error, $\varepsilon$, will sometimes not have uncorrelated elements. This can cause the covariance matrix of $\varepsilon$ to be singular. To generate a residual with uncorrelated elements, the following Singular Value Decomposition (SVD) of the covariance matrix is made.

$$
\begin{aligned}
& \operatorname{Cov}(\varepsilon)= \\
&=\left(\begin{array}{ll}
U_{r} & \tilde{U}_{r}
\end{array}\right)\left(\begin{array}{cc}
\Sigma & 0 \\
0 & 0
\end{array}\right)\left(\begin{array}{c}
U_{r}^{T} \\
\tilde{U}_{r}^{T}
\end{array}\right)= \\
&=U_{r} \Sigma U_{r}^{T}
\end{aligned}
$$

Then the residual

$$
r=U_{r}^{T} \varepsilon,
$$

will have the covariance

$$
\operatorname{Cov}(r)=\mathrm{E} r r^{T}=\mathrm{E} U_{r}^{T} \varepsilon \varepsilon^{T} U_{r}=\Sigma,
$$

which is nonsingular.

\section{GLR TEST STATISTICS}

This section first summarizes and then derives the main results. Three different cases are considered, the difference between them is how the initial state, $x$, is treated. In case 1 , estimation is done from the data window. A parity space method is used in case 2 and in case 3 the method in case 1 is combined with an estimate from old data. The results can be summarized in the following algorithm.

1. Compute the residuals. In order to do this, the following covariances have to be known:

$$
\begin{aligned}
& \operatorname{Cov}\left(\hat{x}^{(1)}\right)=P^{(1)} \quad(\text { Given by a Kalman filter }) \\
& \operatorname{Cov}\left(\hat{x}^{(2)}\right)=\left(\mathcal{O}^{T} S^{-1} \mathcal{O}\right)^{-1}=P^{(2)} \\
& \operatorname{Cov}\left(\varepsilon_{t}^{(1)}\right)=W_{1}^{T} S W_{1} \\
& \operatorname{Cov}\left(\varepsilon_{t}^{(2)}\right)=\left(I-\mathcal{P}_{\mathcal{O}}\right) S\left(I-\mathcal{P}_{\mathcal{O}}\right) \\
& \operatorname{Cov}\left(\varepsilon_{t}^{(3)}\right)=W_{3}^{T} S W_{3}+\mathcal{O} P P^{(1)^{-1}} P \mathcal{O}^{T}
\end{aligned}
$$

where

$$
\begin{aligned}
W_{1}^{T} & =I-\mathcal{O}\left(S^{-1 / 2} \mathcal{O}\right)^{\dagger} S^{-1 / 2} \\
W_{3}^{T} & =I-\mathcal{O} P P^{(2)^{-1}}\left(S^{-1 / 2} \mathcal{O}\right)^{\dagger} S^{-1 / 2} \\
P & =\left(P^{(1)^{-1}}+P^{(2)^{-1}}\right)^{-1} .
\end{aligned}
$$

Form the SVD of the covariances according to (9) and then form the residuals as

$$
\begin{aligned}
& \bar{r}_{t}^{(1)}=\underbrace{\Sigma^{(1)^{-1 / 2}} U_{r}^{(1)^{T}} W_{1}^{T}}_{\bar{W}_{1}}\left(Y-H_{u} U\right) \\
& \bar{r}_{t}^{(2)}=\underbrace{\Sigma^{(2)}{ }^{-1 / 2} U_{r}^{(2)^{T}}\left(I-\mathcal{P}_{\mathcal{O}}\right)}_{\bar{W}_{2}}\left(Y-H_{u} U\right) \\
& \left.\bar{r}_{t}^{(3)}=\Sigma_{\Sigma^{(3)^{-1 / 2}} U_{r}^{(3)^{T}}\left(W_{3}^{T}\left(Y-H_{u} U\right)\right.}-\mathcal{O} P P^{(1)^{-1}} \hat{x}^{(1)}\right) .
\end{aligned}
$$

Also define

$$
\bar{W}_{3}=\Sigma^{(3)^{-1 / 2}} U_{r}^{(3)^{T}} W_{3}^{T} .
$$

2. Compute the test statistics $L_{i, c}$ and $L_{i, r}$ where $i$ indicates the method used (case $i$ ) and $\mathrm{c}, \mathrm{r}$ indicates conventional or robust GLR test respectively.

$$
\begin{aligned}
L_{1, c}= & L_{1, r}=\bar{r}_{t}^{(1) T} \mathcal{P}_{\bar{W}_{1}^{T} H_{f}} \bar{r}_{t}^{(1)} \\
L_{2, c}= & L_{2, r}=\bar{r}_{t}^{(2) T} \mathcal{P}_{\bar{W}_{2}^{T} H_{f}} \bar{r}_{t}^{(2)} \\
L_{3, c} & =\bar{r}_{t}^{(3) T} \mathcal{P}_{\bar{W}_{3}^{T} H_{f}} \bar{r}_{t}^{(3)} \\
L_{3, r} & =\bar{r}_{t}^{(3) T} \mathcal{P}_{\bar{W}_{3}^{T}\left(I-\mathcal{P}_{\mathcal{O}}\right) H_{f}} \bar{r}_{t}^{(3)}
\end{aligned}
$$

3. Compute thresholds for detecting faults. All thresholds are $\chi^{2}\left(\operatorname{rank}\left(\mathcal{P}_{A}\right)\right)$-distributed, where $\mathcal{P}_{A}$ denotes the projection that is used to compute the test statistic. See also section 2.2.

\subsection{Case 1: state estimation in sliding window}

In this case, $x$ is estimated by minimum variance estimation from data in the time window as

$$
\begin{aligned}
& \hat{x}^{(2)}=\left(S^{-1 / 2} \mathcal{O}\right)^{\dagger} S^{-1 / 2}\left(Y-H_{u} U\right) \\
\sim & \mathrm{N}\left(x+\left(S^{-1 / 2} \mathcal{O}\right)^{\dagger} S^{-1 / 2} H_{f} F,\left(\mathcal{O}^{T} S^{-1} \mathcal{O}\right)^{-1}\right),
\end{aligned}
$$

then the prediction error becomes

$$
\begin{array}{r}
\varepsilon_{t}^{(1)}=Y-H_{u} U-\mathcal{O} \hat{x}^{(2)}= \\
\underbrace{\left(I-\mathcal{O}\left(S^{-1 / 2} \mathcal{O}\right)^{\dagger} S^{-1 / 2}\right)}_{W_{1}^{T}}\left(Y-H_{u} U\right) \\
\sim \mathrm{N}\left(W_{1}^{T} H_{f} F, \operatorname{Cov}\left(\varepsilon_{t}^{(1)}\right)\right) .
\end{array}
$$

The covariance becomes

$$
\operatorname{Cov}\left(\varepsilon_{t}^{(1)}\right)=W_{1}^{T} S W_{1} .
$$

To form a residual with uncorrelated elements, an SVD is formed according to (9) as

$$
\operatorname{Cov}\left(\varepsilon_{t}^{(1)}\right)=U_{r}^{(1)} \Sigma^{(1)} U_{r}^{(1)^{T}} .
$$


Then the residual becomes

$$
r_{t}^{(1)}=U_{r}^{(1)^{T}} \varepsilon_{t}^{(1)} .
$$

To determine the likelihood ratio with a hypothesis test, a normalized residual is formed as

$$
\begin{aligned}
\bar{r}_{t}^{(1)}= & \left(\operatorname{Cov}\left(r_{t}^{(1)}\right)\right)^{-1 / 2} r_{t}^{(1)} \\
= & \underbrace{\Sigma^{(1)^{-1 / 2}} U_{r}^{(1)^{T}} W_{1}^{T}}_{\bar{W}_{1}^{T}}\left(Y-H_{u} U\right) \\
& \sim \mathrm{N}\left(\bar{W}_{1}^{T} H_{f} F, I\right) .
\end{aligned}
$$

Conventional GLR Test The hypotheses in (2) are here

$$
\begin{array}{ll}
H_{0}: & \bar{r}_{t}^{(1)} \sim \mathrm{N}(0, I) \\
H_{1}: & \bar{r}_{t}^{(1)} \sim \mathrm{N}\left(\bar{W}_{1}^{T} H_{f} F, I\right) .
\end{array}
$$

This gives the log-likelihood ratio

$$
L_{1, c}=2 \max _{F} \log \frac{e^{-\frac{1}{2}\left\|\bar{r}_{t}^{(1)}-\bar{W}_{1}^{T} H_{f} F\right\|_{2}^{2}}}{e^{-\frac{1}{2}\left\|\bar{r}_{t}^{(1)}\right\|_{2}^{2}}},
$$

which is maximized when $F=\left(\bar{W}_{1}^{T} H_{f}\right)^{\dagger} \bar{r}_{t}^{(1)}$. Then,

$$
\begin{aligned}
& L_{1, c}= \\
& -\|\bar{r}_{t}^{(1)}-\underbrace{\bar{W}_{1}^{T} H_{f}\left(\bar{W}_{1}^{T} H_{f}\right)^{\dagger}}_{\mathcal{P}_{\bar{W}_{1}^{T} H_{f}}} \bar{r}_{t}^{(1)}\|_{2}^{2}+\left\|\bar{r}_{t}^{(1)}\right\|_{2}^{2} \\
& =-\bar{r}_{t}^{(1) T}\left(I-\mathcal{P}_{\bar{W}_{1}^{T} H_{f}}\right)^{T}\left(I-\mathcal{P}_{\bar{W}_{1}^{T} H_{f}}\right) \bar{r}_{t}^{(1)} \\
& \quad+\bar{r}_{t}^{(1) T} \bar{r}_{t}^{(1)}=\bar{r}_{t}^{(1) T} \mathcal{P}_{\bar{W}_{1}^{T} H_{f}} \bar{r}_{t}^{(1)}= \\
& =\left\|\bar{r}_{t}^{(1)}\right\|_{\mathcal{P}_{\bar{W}_{1}^{T} H_{f}}^{2}}^{2}
\end{aligned}
$$

Robust GLR Test The hypotheses in the robust test questions if there is no fault or if the fault resides in the subspace orthogonal to the signal space. Thus, the hypotheses in (3) are

$$
\begin{array}{ll}
H_{0}: & \bar{r}_{t}^{(1)} \sim \mathrm{N}(0, I) \\
H_{1}: & \bar{r}_{t}^{(1)} \sim \mathrm{N}\left(\bar{W}_{1}^{T}\left(I-\mathcal{P}_{\mathcal{O}}\right) H_{f} F, I\right) .
\end{array}
$$

The log-likelihood ratio becomes

$$
L_{1, r}=2 \max _{F} \log \frac{e^{-\frac{1}{2}\left\|\bar{r}_{t}^{(1)}-\bar{W}_{1}^{T}\left(I-\mathcal{P}_{\mathcal{O}}\right) H_{f} F\right\|_{2}^{2}}}{e^{-\frac{1}{2}\left\|\bar{r}_{t}^{(1)}\right\|_{2}^{2}}} .
$$

This ratio is maximized for

$$
F=\left(\bar{W}_{1}^{T}\left(I-\mathcal{P}_{\mathcal{O}}\right) H_{f}\right)^{\dagger} \bar{r}_{t}^{(1)}
$$

which gives

$$
L_{1, r}=\bar{r}_{t}^{(1) T} \mathcal{P}_{\bar{W}_{1}^{T}\left(I-\mathcal{P}_{\mathcal{O}}\right) H_{f}} \bar{r}_{t}^{(1)}
$$

Lemma 1. The robust and conventional tests for case 1 coincide since

$$
\bar{W}_{1}^{T}=\bar{W}_{1}^{T}\left(I-\mathcal{P}_{\mathcal{O}}\right) .
$$

PROOF. Since

$$
\bar{W}_{1}^{T}=\Sigma^{(1)^{-1 / 2}} U_{r}^{(1)^{T}} W_{1}^{T},
$$

it is sufficient to prove that $W_{1}^{T} \mathcal{P}_{\mathcal{O}}=0$ to justify (22).

$$
\begin{aligned}
W_{1}^{T} \mathcal{P}_{\mathcal{O}} & =\left(I-\mathcal{O}\left(S^{-1 / 2} \mathcal{O}\right)^{\dagger} S^{-1 / 2}\right) \mathcal{O} \mathcal{O}^{\dagger} \\
& =\mathcal{O}(I-\underbrace{\left(S^{-1 / 2} \mathcal{O}\right)^{\dagger} S^{-1 / 2} \mathcal{O}}_{=I}) \mathcal{O}^{\dagger}=0
\end{aligned}
$$

Then, according to Lemma 1 , the robust test statistic can be written as

$$
L_{1, r}=\bar{r}_{t}^{(1) T} \mathcal{P}_{\bar{W}_{1}^{T} H_{f}} \bar{r}_{t}^{(1)}=\left\|\bar{r}_{t}^{(1)}\right\|_{\mathcal{P}_{\bar{W}_{1}^{T} H_{f}}^{2}}^{2} .
$$

\subsection{Case 2: parity space approach}

In this case the residual, $Y-H_{u} U$, is multiplied with the orthogonal complement of $\mathcal{O}, I-\mathcal{P}_{\mathcal{O}}$, to eliminate the dependence on $x$. Then the prediction error becomes

$$
\begin{aligned}
\varepsilon_{t}^{(2)}=\underbrace{\left(I-\mathcal{P}_{\mathcal{O}}\right)}_{W_{2}^{T}}( & \left.Y-H_{u} U\right) \\
& \sim \mathrm{N}\left(W_{2}^{T} H_{f} F, W_{2}^{T} S W_{2}\right) .
\end{aligned}
$$

A residual with uncorrelated elements is formed by (10) as

$$
W_{2}^{T} S W_{2}=U_{r}^{(2)} \Sigma^{(2)} U_{r}^{(2)^{T}} \Rightarrow r_{t}^{(2)}=U_{r}^{(2)^{T}} \varepsilon_{t}^{(2)} .
$$

In order to get unit variance, the residual can be normalized as

$$
\begin{aligned}
\bar{r}_{t}^{(2)}= & \Sigma^{(2)^{-1 / 2} r_{t}^{(2)}} \\
= & \underbrace{\Sigma^{(2)}{ }^{-1 / 2} U_{r}^{(2)^{T}} W_{2}^{T}}_{\bar{W}_{2}^{T}}\left(Y-H_{u} U\right) \\
& \sim \mathrm{N}\left(\bar{W}_{2}^{T} H_{f} F, I\right) .
\end{aligned}
$$

Conventional GLR Test The hypotheses in (2) are here

$$
\begin{array}{ll}
H_{0}: & \bar{r}_{t}^{(2)} \sim \mathrm{N}(0, I) \\
H_{1}: & \bar{r}_{t}^{(2)} \sim \mathrm{N}\left(W_{2}^{T} H_{f} F, I\right) .
\end{array}
$$

This gives the log-likelihood ratio fault/no fault

$$
\begin{aligned}
& L_{2, c}=2 \max _{F} \log \frac{e^{-\frac{1}{2}\left\|\bar{r}_{t}^{(2)}-\bar{W}_{2}^{T} H_{f} F\right\|_{2}^{2}}}{e^{-\frac{1}{2}\left\|\bar{r}_{t}^{(2)}\right\|_{2}^{2}}}= \\
& =\max _{F}-\left(\left\|\bar{r}_{t}^{(2)}-\bar{W}_{2}^{T} H_{f} F\right\|_{2}^{2}-\left\|\bar{r}_{t}^{(2)}\right\|_{2}^{2}\right)
\end{aligned}
$$

This ratio is maximized when $F=\left(\bar{W}_{2}^{T} H_{f}\right)^{\dagger} \bar{r}_{t}^{(2)}$, then the expression becomes 


$$
\begin{aligned}
& L_{2, c}=-\|\bar{r}_{t}^{(2)}-\underbrace{\bar{W}_{2}^{T} H_{f}\left(\bar{W}_{2}^{T} H_{f}\right)^{\dagger}}_{\mathcal{P}_{\bar{W}_{2}^{T} H_{f}}} \bar{r}_{t}^{(2)}\|_{2}^{2} \\
& +\left\|\bar{r}_{t}^{(2)}\right\|_{2}^{2}=\bar{r}_{t}^{(2) T} \mathcal{P}_{\bar{W}_{2}^{T} H_{f}} \bar{r}_{t}^{(2)}=\left\|\bar{r}_{t}^{(2)}\right\|_{\mathcal{P}_{\bar{W}_{2}^{T} H_{f}}}^{2} .
\end{aligned}
$$

Robust GLR Test Also for this case the robust hypotheses derived from (3) becomes

$$
\begin{array}{ll}
H_{0}: & \bar{r}_{t}^{(2)} \sim \mathrm{N}(0, I) \\
H_{1}: & \bar{r}_{t}^{(2)} \sim \mathrm{N}\left(W_{2}^{T}\left(I-\mathcal{P}_{\mathcal{O}}\right) H_{f} F, I\right) .
\end{array}
$$

When calculating the test statistic as in section 3.1 , it becomes

$$
L_{2, r}=\left\|\bar{r}_{t}^{(2)}\right\|_{\mathcal{P}_{\bar{W}_{2}^{T} H_{f}}}^{2} .
$$

This is the same residuals that we are testing with the conventional test. So when estimating $x$ by projection, the robust and conventional tests coincide. The reason for this is that the the term $I-\mathcal{P}_{\mathcal{O}}$ is already a part of $W_{2}^{T}$ and $\left(I-\mathcal{P}_{\mathcal{O}}\right)\left(I-\mathcal{P}_{\mathcal{O}}\right)=I-\mathcal{P}_{\mathcal{O}}$.

\subsection{Case 3: state estimation in preceding and sliding} window

In this case $x$ is estimated by a minimum variance estimator $\hat{x}^{(2)}$ with covariance $P^{(2)}$ from data in the sliding window as in section 3.1, but also with a Kalman filter from old data, providing $\hat{x}^{(1)}$ with covariance matrix $P^{(1)}$. This appears to be a logical approach to detect faults in the signal space, since the difference in state estimates should be due to estimation errors and faults in the signal space only. For instance, we have

$$
\begin{aligned}
r_{t}=\mathcal{O} & \left(\hat{x}^{(2)}-\hat{x}^{(1)}\right) \\
& \sim \mathrm{N}\left(\mathcal{O}\left(S^{-1 / 2} \mathcal{O}\right)^{\dagger} S^{-1 / 2} H_{f} F_{t},\right. \\
& \left.\mathcal{O}\left(P^{(1)}+P^{(2)}\right) \mathcal{O}^{T}\right) .
\end{aligned}
$$

This is a residual for testing faults in the signal space. For faults orthogonal to the signal space, we proceed by forming the joint state estimate over all data by the standard sensor fusion formula as outlined below. The estimate from the Kalman filter is assumed to be Gaussian distributed

$$
\hat{x}^{(1)} \sim \mathrm{N}\left(x, P^{(1)}\right) .
$$

The minimum variance estimate of $x$ from data in the window is given by

$$
\begin{array}{r}
\hat{x}^{(2)}=\left(S^{-1 / 2} \mathcal{O}\right)^{\dagger} S^{-1 / 2}\left(Y-H_{u} U\right) \\
\sim \mathrm{N}\left(x+\left(S^{-1 / 2} \mathcal{O}\right)^{\dagger} S^{-1 / 2} H_{f} F,\right. \\
\underbrace{\left(\mathcal{O}^{T} S^{-1} \mathcal{O}\right)^{-1}}_{P(2)}) .
\end{array}
$$

Then,

$$
\hat{x}=P\left(P^{(1)^{-1}} \hat{x}^{(1)}+P^{(2)^{-1}} \hat{x}^{(2)}\right),
$$

where

$$
P=\left(P^{(1)^{-1}}+P^{(2)^{-1}}\right)^{-1} .
$$

The prediction error is then formed as

$$
\begin{aligned}
& \varepsilon_{t}^{(3)}= Y-H_{u} U-\mathcal{O} \hat{x} \\
&= \underbrace{\left(I-\mathcal{O} P P^{(2)^{-1}}\left(S^{-1 / 2} \mathcal{O}\right)^{\dagger} S^{-1 / 2}\right)}_{W_{3}^{T}} \times \\
& \times\left(Y-H_{u} U\right)-\mathcal{O} P P^{(1)^{-1}} \hat{x}^{(1)} \\
&=W_{3}^{T}\left(\mathcal{O} x+H_{f} F+E\right)-\mathcal{O} P P^{(1)^{-1}}\left(x+\tilde{x}^{(1)}\right) \\
&=\mathcal{O} x-\underbrace{\left(\mathcal{O} P P^{(2)^{-1}} x+\mathcal{O} P P^{(1)^{-1}} x\right)}_{\mathcal{O} x} \\
&+W_{3}^{T}\left(H_{f} F+H_{v} V+E\right)-P P^{(1)}{ }^{-1} \tilde{x}^{(1)} \\
&=W_{3}^{T}\left(H_{f} F+E\right)-P P^{(1)^{-1}} \tilde{x}^{(1)} \\
& \sim \mathrm{N}\left(W_{3}^{T} H_{f} F, \operatorname{Cov}\left(\varepsilon_{t}^{(3)}\right)\right)
\end{aligned}
$$

where

$$
\begin{gathered}
\tilde{x}^{(1)}=\hat{x}^{(1)}-x \sim \mathrm{N}\left(0, P^{(1)}\right) \\
\operatorname{Cov}\left(\varepsilon_{t}^{(3)}\right)=W_{3}^{T} S W_{3}+\mathcal{O} P P^{(1)^{-1}} P \mathcal{O}^{T} .
\end{gathered}
$$

For the same reasons as in section 3.1 and 3.2 , an SVD is formed of the covariance matrix to get a basis for a residual with uncorrelated elements.

$$
\left.\operatorname{Cov}\left(\varepsilon_{t}^{(3)}\right)\right)=U_{r}^{(3)} \Sigma^{(3)} U_{r}^{(3)^{T}} \Rightarrow r_{t}^{(3)}=U_{r}^{(3)^{T}} \varepsilon_{t}^{(3)}
$$

The normalized residual is then formed as

$$
\begin{aligned}
\bar{r}_{t}^{(3)}=\Sigma^{(3)} & -1 / 2 r_{t}^{(3)} \\
& \sim \mathrm{N}(\underbrace{\Sigma^{(3)^{-1 / 2}} U_{r}^{(3)^{T}} W_{3}^{T}}_{\bar{W}_{3}^{T}} H_{f} F, I) .
\end{aligned}
$$

Conventional Likelihood Test The hypotheses for the conventional test are

$$
\begin{aligned}
& H_{0}: \bar{r}_{t}^{(3)} \sim \mathrm{N}(0, I) \\
& H_{1}: \bar{r}_{t}^{(3)} \sim \mathrm{N}\left(\bar{W}_{3}^{T} H_{f} F, I\right) .
\end{aligned}
$$

This yields the log-likelihood ratio

$$
\begin{aligned}
& L_{3, c}=\max _{F} \log \frac{e^{-\frac{1}{2}\left\|\bar{r}_{t}^{(3)}-\bar{W}_{3}^{T} H_{f} F\right\|_{2}^{2}}}{e^{-\frac{1}{2}\left\|\bar{r}_{t}^{(3)}\right\|_{2}^{2}}}= \\
& =\max _{F}-\left(\left\|\bar{r}_{t}^{(3)}-\bar{W}_{3}^{T} H_{f} F\right\|_{2}^{2}-\left\|\bar{r}_{t}^{(3)}\right\|_{2}^{2}\right) .
\end{aligned}
$$

Which is maximized for $F=\left(\bar{W}_{3}^{T} H_{f}\right)^{\dagger} \bar{r}_{t}^{(3)}$, then

$$
\begin{aligned}
& L_{3, c}= \\
& =-\left(\left\|(I-\underbrace{\bar{W}_{3}^{T} H_{f}\left(\bar{W}_{3}^{T} H_{f}\right)^{\dagger}}_{\mathcal{P}_{\bar{W}_{3}^{T} H_{f}}}) \bar{r}_{t}^{(3)}\right\|_{2}^{2}-\left\|\bar{r}_{t}^{(3)}\right\|_{2}^{2}\right) \\
& \quad=\bar{r}_{t}^{(3) T} \mathcal{P}_{\bar{W}_{3}^{T} H_{f}} \bar{r}_{t}^{(3)}=\left\|\bar{r}_{t}^{(3)}\right\|_{\mathcal{P}_{\bar{W}_{3}^{T} H_{f}}^{2}}^{2}
\end{aligned}
$$


Robust Likelihood Test The hypotheses for the robust test are

$$
\begin{aligned}
& H_{0}: \bar{r}_{t}^{(3)} \sim \mathrm{N}(0, I) \\
& H_{1}: \bar{r}_{t}^{(3)} \sim \mathrm{N}\left(\bar{W}_{3}^{T}\left(I-\mathcal{P}_{\mathcal{O}}\right) H_{f} F, I\right) .
\end{aligned}
$$

Similar calculations as for the conventional test yield the robust log-likelihood ratio

$$
\begin{aligned}
L_{3, r}=\bar{r}_{t}^{(3) T} \mathcal{P}_{\bar{W}_{3}^{T}\left(I-\mathcal{P}_{\mathcal{O}}\right) H_{f}} \bar{r}_{t}^{(3)}= \\
\quad=\left\|\bar{r}_{t}^{(3)}\right\|_{\mathcal{P}_{\bar{W}_{3}^{T}\left(I-\mathcal{P}_{\mathcal{O}}\right) H_{f}}^{2}}^{2} .
\end{aligned}
$$

Comments on the Tests These tests are closely related to case 1 where $x$ is determined only from data in the window. If $P^{(1)} \rightarrow \infty I$, then the normalized residual in (39) is identical to the one in case 1 and therefore also the detector.

Consider the case where $P^{(2)} \rightarrow \infty I$. Then it is easy to see that $W_{3}^{T}=I$ and $P=P^{(1)}$. The prediction error is then

$$
\begin{gathered}
\varepsilon_{t}^{(3)}=Y-H u U-\mathcal{O} \hat{x}^{(1)} \sim N\left(H_{f} F, \operatorname{Cov}\left(\varepsilon_{t}^{(3)}\right)\right) \\
\operatorname{Cov}\left(\varepsilon_{t}^{(3)}\right)=S+\mathcal{O} P^{(1)} \mathcal{O}^{T} .
\end{gathered}
$$

Since $S$ has full rank, $\operatorname{Cov}\left(\varepsilon_{t}^{(3)}\right)$ will have full rank. Therefore, no dimension reduction has to be done when forming the residual. The normalized residual then becomes

$$
\bar{r}_{t}^{(3)}=\operatorname{Cov}\left(\varepsilon_{t}^{(3)}\right)^{-1 / 2}\left(Y-H_{u} U-\mathcal{O} \hat{x}^{(1)}\right) .
$$

Lemma 2. If the uncertainty in $\hat{x}^{(1)}$ is large, i.e., $P^{(1)} \rightarrow \infty I$, then the normalized residual in (45) will not be affected by signals in the signal space. Thus, the test coincides with case 2 , where the signal space is projected away.

PROOF. Rewrite the covariance matrix of the prediction error as

$$
\begin{aligned}
& \operatorname{Cov}\left(\varepsilon_{t}^{(3)}\right)=S+\mathcal{O} P^{(1)} \mathcal{O}^{T}= \\
& S+\left(\begin{array}{ll}
\mathcal{O} & \mathcal{N}_{\mathcal{O}}
\end{array}\right)\left(\begin{array}{cc}
P^{(1)} & 0 \\
0 & 0
\end{array}\right)\left(\begin{array}{c}
\mathcal{O}^{T} \\
\mathcal{N}_{\mathcal{O}}^{T}
\end{array}\right)
\end{aligned}
$$

where $\mathcal{N}_{\mathcal{O}}$ is a basis of the orthogonal complement to $\mathcal{O}$. The matrix $\left(\mathcal{O} \mathcal{N}_{\mathcal{O}}\right)$ thus span the whole space for $\operatorname{Cov}\left(\varepsilon_{t}^{(3)}\right)$. We can therefore write

$$
S=\left(\begin{array}{ll}
\mathcal{O} & \mathcal{N}_{\mathcal{O}}
\end{array}\right)\left(\begin{array}{cc}
T & 0 \\
0 & \tilde{T}
\end{array}\right)\left(\begin{array}{c}
\mathcal{O}^{T} \\
\mathcal{N}_{\mathcal{O}}{ }^{T}
\end{array}\right),
$$

where $T$ and $\tilde{T}$ is chosen suitably. Then,

$$
\begin{aligned}
& \operatorname{Cov}\left(\varepsilon_{t}^{(3)}\right)^{-1 / 2}= \\
& \left(\mathcal{O} \mathcal{N}_{\mathcal{O}}\right)\left(\begin{array}{cc}
\left(T+P^{(1)}\right)^{-1 / 2} & 0 \\
0 & \tilde{T}^{-1 / 2}
\end{array}\right)\left(\begin{array}{c}
\mathcal{O}^{T} \\
\mathcal{N}_{\mathcal{O}}{ }^{T}
\end{array}\right)
\end{aligned}
$$

and when the uncertainty in $\hat{x}^{(1)}$ is large then

$$
\begin{aligned}
\lim _{P^{(1) \rightarrow \infty I}} \operatorname{Cov}\left(\varepsilon_{t}^{(3)}\right)^{-1 / 2}= \\
\left(\begin{array}{lll}
\mathcal{O} & \mathcal{N}_{\mathcal{O}}
\end{array}\right)\left(\begin{array}{cc}
0 & 0 \\
0 & \tilde{T}^{-1 / 2}
\end{array}\right)\left(\begin{array}{c}
\mathcal{O}^{T} \\
\mathcal{N}_{\mathcal{O}}{ }^{T}
\end{array}\right)= \\
\mathcal{N}_{\mathcal{O}} \tilde{T}^{-1 / 2} \mathcal{N}_{\mathcal{O}}{ }^{T},
\end{aligned}
$$

which is a projection on the space orthogonal to $\mathcal{O}$.

\section{CONCLUSIONS}

The original generalized likelihood ratio (GLR) test for fault detection where derived using a Kalman filter approach, and thus all past data influence the test statistic. On the other hand, the parity space approach is defined over a sliding window. In order to compare these standard approaches, we have derived the GLR test statistic for fault detection in linear Gaussian systems based on data over a sliding window. This is done in a systematic way for different assumption on the fault range space (robust/non-robust) and residual generators (parity space/state estimation). It turns out that all these cases correspond to different projections of the basic model residual.

\section{REFERENCES}

Basseville, M. and I. V. Nikiforov (1993). Detection of Abrupt Changes, Theory and Application. Information and system sciences series. Prentice-Hall. Enlewood Cliffs, NJ.

Chow, A. Y. and A. S. Willsky (1984). Analytical redundancy and the design of robust failure detection systems. IEEE Transactions on Automatic Control 29(7), 603-614.

Desai, M. N. and R. S. Mangoubi (2003). Robust gaussian and non-gaussian matched subspace detection. IEEE Transactions on Signal Processing 51(12), 3115-3127.

Ding, X., L. Guo and T. Jeinsch (1999). A characterization of parity space and its application to robust fault detection. IEEE Transactions on Automatic Control 44(2), 337-343.

Gertler, J. (1997). Fault detection and isolation using parity relations. Control Engineering Practice 5(5), 653-661.

Gertler, J. J. (1998). Fault Detection and Diagnosis in Engineering Systems. Marcel Dekker, Inc.

Golub, G. H. and C. F. van Loan (1996). Matrix Computations. 3 ed. John Hopkins University Press.

Gustafsson, F. (2001). Adaptive filtering and change detection. John Wiley \& Sons, Inc.

Gustafsson, F. (2002). Stochastic fault diagnosability in parity spaces. In: Proceedings of the 15th IFAC World Congress. Barcelona, Spain.

Willsky, A. and H. Jones (1976). A generalized likelihood ratio approach to the detection and estimation of jumps in linear systems. IEEE Transactions on Automatic Control 21, 108-112. 